\title{
A generalised combinatorial distribution problem
}

\author{
By G. Baikunth NATH \\ (Received March 17, 1972) \\ (Revised Aug. 3, 1972)
}

\section{$\S 1$. Introduction and summary.}

Let $A=\left(a_{i j}\right)$ be a square matrix of size $n$ and let the entries of $A$ be non-negative integers. Denote the sum of row $i$ of $A$ by $r_{i}, r_{i} \geqq 0$, and that of the column $j$ of $A$ by $s_{j}, s_{j} \geqq 0$. If $T$ denotes the total sum in $A$, then it is clear that

$$
T=\sum_{i=1}^{n} r_{i}=\sum_{j=1}^{n} s_{j}
$$

We call $R=\left(r_{1}, r_{2}, \cdots, r_{n}\right)$ the row sum vector and $S=\left(s_{1}, s_{2}, \cdots, s_{n}\right)$ the column sum vector of $A$. The vectors $R$ and $S$ determine a class

$$
G=G(R, S),
$$

consisting of all such matrices of size $n$, with row sum vector $R$ and column sum vector $S$. For $A$ admitting integers 0 and 1 only, known as $(0,1)$-matrix, many diversified topics including traces, term ranks, widths, heights, and combinatorial designs related to problems dealing with a class $G^{\prime}$, a subclass of $G$, consisting of $(0,1)$-matrices, have attracted the attention of many authors. Among them are Ryser (1957, 1960a, 1960b), Jurkat and Ryser (1967), and Murty (1968). A detailed list of references may be found in Ryser (1960a).

Let $H(n, R, S)$ denote the number of members of class $G$, that is the number of ways in which $n$ distinct things, the $j$-th replicated $s_{j}$ times, $s_{j} \geqq 0$, can be distributed among $n$ persons, the $i$-th getting $r_{i}, r_{i} \geqq 0$. The case, when each row sum and column sum equals $r(\geqq 1)$, and the number $H(n, R, R)$ denoted by $H(n, r)$, has been investigated by Kenji Mano (1961). He gives an intricate formula for $r=2$. Anand et al. (1966) extended the result to $H(3, r)$ and stated a plausible formula for $H(n, r)$. Recently, Nath and Iyer (1972) have suggested the use of the generating functions to expedite calculations and obtained explicit formulae for $H(3, r)$ and $H(4, r)$.

In the present paper, we give some inequalities for $H(n, R, S)$, true for all positive $n$, and an explicit formula for $H(3, R, S)$. The procedure applies to rectangular matrices as well as square ones. 
§ 2. Inequalities for $H(n, R, S)$.

If we take $a_{i j}$ to denote the number of objects of the $j$-th type which are given to the person $i$, in any distribution, then it is readily seen that $H(n, R, S)$ is the number of matrices in class $G(R, S)$.

We, now, proceed to show that $H(n, R, S)$ is the number of ways in which $s_{1}$ ones, $s_{2}$ twos, $s_{3}$ threes, $\cdots, s_{n} n$ 's can be written in $n$ rows, $r_{i}$ in the $i$-th row, the order of elements in any row being immaterial.

This follows at once if we take the elements in the $i$-th row to denote the objects which are given to the person $i$. If $j$ occurs $k$ times in the $i$-th row, it is taken to mean that $i$ gets $k$ objects of the type $j$.

If the order of elements in each row were relevant, then the number of ways of writing $s_{1}+s_{2}+\cdots+s_{n}$ objects $1,2, \cdots, n$, the $j$-th repeated $s_{j}$ times, in $n$ rows, $r_{i}$ in the $i$-th row, would be $\left(s_{1}+s_{2}+\cdots+s_{n}\right) ! /\left(r_{1} ! r_{2} ! \cdots r_{n} !\right)$.

Hence

$$
H(n, R, S) \leqq\left(\Sigma s_{i}\right) ! / \Pi\left(r_{i} !\right), \quad i=1,2, \cdots, n .
$$

Since the $r_{i}$ elements in the $i$-th row can be arranged among themselves in at most $r_{i}$ ! ways, we must have

$$
H(n, R, S) \geqq\left(\sum s_{i}\right) ! / \Pi\left(r_{i} !\right)^{2} .
$$

\section{§3. Reduction of the problem.}

As stated earlier, for $n=3, H(3, R, S)$ is the number of matrices

$$
\left[\begin{array}{lll}
a_{11} & a_{12} & a_{13} \\
a_{21} & a_{22} & a_{23} \\
a_{31} & a_{32} & a_{33}
\end{array}\right], \quad a_{i j} \geqq 0,
$$

such that the row and column sums respectively are $r_{i}$ and $s_{i}, i=1,2,3$.

If $\left(r_{1}, r_{2}, r_{3}\right)$ and $\left(s_{1}, s_{2}, s_{3}\right)$ are, in order, allowing row and column permutations, we can assume that

and

$$
0 \leqq r_{3} \leqq r_{2} \leqq r_{1}
$$

$$
0 \leqq s_{3} \leqq s_{2} \leqq s_{1}
$$

Further assume that $r_{1}>s_{1}$, in case they are equal take $r_{2} \geqq s_{2}$. Let $M(3, R, S)$, then, denote the number of matrices with row and column sums, in order.

Let $N_{1}\left(r_{1}, r_{2}, r_{3}\right)$ and $N_{2}\left(s_{1}, s_{2}, s_{3}\right)$ respectively denote the number of ways in which the numbers $\left(r_{1}, r_{2}, r_{3}\right)$ and $\left(s_{1}, s_{2}, s_{3}\right)$ can be permuted among themselves. That is 


$$
\begin{aligned}
N_{1}\left(r_{1}, r_{2}, r_{3}\right) & =1, & & \text { if } r_{1}=r_{2}=r_{3} \\
& =6, & & \text { if } r_{1} \neq r_{2} \neq r_{3} \\
& =3, & & \text { otherwise. }
\end{aligned}
$$

Similar relations holds for $N_{2}\left(s_{1}, s_{2}, s_{3}\right)$.

Then

$$
H(3, R, S)=N_{1}\left(r_{1}, r_{2}, r_{3}\right) N_{2}\left(s_{1}, s_{2}, s_{3}\right) M(3, R, S) .
$$

\section{$\S 4$. An expression for $M$.}

We assert that

$$
\begin{aligned}
M(3, R, S)= & \left(\begin{array}{c}
r_{2}+2 \\
2
\end{array}\right)\left\{\left(\begin{array}{c}
r_{3}+2 \\
2
\end{array}\right)-\Sigma\left(\begin{array}{c}
r_{3}-s_{i}+1 \\
2
\end{array}\right)\right\} \\
& -\Sigma\left\{\left(\begin{array}{c}
r_{2}+r_{3}-s_{i}+3 \\
4
\end{array}\right)-\left(\begin{array}{c}
r_{2}-s_{i}+2 \\
4
\end{array}\right)\right. \\
& \left.-\left(r_{3}+1\right)\left(\begin{array}{c}
r_{2}-s_{i}+1 \\
3
\end{array}\right)\right\},
\end{aligned}
$$

the summations extending over $i=1,2,3$.

PROOF. Let the entries in the first row $\left(a_{1}, a_{2}, a_{3}\right)$ be arbitrarily fixed, then it remains to arrange the remaining $s_{i}-a_{i}(i=1,2,3)$ elements in the last two rows of the three columns respectively.

The generating function for such a scheme is

$$
\Pi\left(1-x^{s_{i-a_{i}+1}}\right)(1-x)^{-3} .
$$

The coefficient of $x^{r_{2}}$ in (4.2) namely,

$$
\left(\begin{array}{c}
r_{2}+2 \\
2
\end{array}\right)-\sum_{i=1}^{3}\left(\begin{array}{c}
r_{2}-s_{i}+a_{i}+1 \\
2
\end{array}\right)
$$

gives the number of arrangements for row and column sums equal to $r_{i}$ and $s_{i}$ respectively, conditional on the specification that the row one has entries $\left(a_{1}, a_{2}, a_{3}\right)$. The value $M(3, R, S)$ then results from summing (4.3) over all non-negative integral triples $\left(a_{1}, a_{2}, a_{3}\right)$ compatible with $a_{1}+a_{2}+a_{3}=r_{1}$.

Since the first term in (4.3) is constant and does not depend on the entries $\left(a_{1}, a_{2}, a_{3}\right)$ of the specified row one, its sum over non-negative integral values is achieved by multiplying with the coefficient of $x^{r_{3}}$ in the generating function

namely

$$
\Pi\left(1-x^{s_{i+1}}\right)(1-x)^{-3},
$$

$$
\left(\begin{array}{c}
r_{2}+2 \\
2
\end{array}\right)\left\{\left(\begin{array}{c}
r_{3}+2 \\
2
\end{array}\right)-\Sigma\left(\begin{array}{c}
r_{3}-s_{i}+1 \\
2
\end{array}\right)\right\}, \quad i=1,2,3
$$


Next, the sum of the second term of (4.3) is conditional with respect to the entries of the first row. Its product with the coefficient of $x^{r_{3}-a_{i}}$ in the generating function

$$
\Pi\left(1-x^{s_{k+1}}\right)(1-x)^{-2},
$$

$k=1,2,3$ and different from $i$, namely

$$
\begin{aligned}
& \sum_{a_{i}=0}^{s i}\left(\begin{array}{c}
r_{2}-s_{i}+a_{i}+1 \\
2
\end{array}\right)\left(r_{3}-s_{i}+1\right), \quad i=1,2,3, \\
= & \sum_{i=1}^{3}\left\{\left(\begin{array}{c}
r_{2}+r_{3}-s_{i}+3 \\
4
\end{array}\right)-\left(\begin{array}{c}
r_{2}-s_{i}+2 \\
4
\end{array}\right)-\left(r_{3}+1\right)\left(\begin{array}{c}
r_{2}-s_{i}+1 \\
3
\end{array}\right)\right\}
\end{aligned}
$$

gives the sum.

Subtraction of (4.6) from (4.5) determines the number $M(3, R, S)$. Hence the assertion.

An expression similar to (4.1) but simpler in form, for the $(0,1)$-matrices, can be obtained much on the same lines, and is left to the reader.

EXAMPLE 1. Let $r_{1}=6, r_{2}=4, r_{3}=2, s_{1}=5, s_{2}=4$, and $s_{3}=3$, then we have

and hence

$$
\begin{aligned}
& N_{1}\left(r_{1}, r_{2}, r_{3}\right)=6, \\
& N_{2}\left(s_{1}, s_{2}, s_{3}\right)=6, \\
& M(3, R, S)=69 \quad \text { from }(4.1),
\end{aligned}
$$

$$
H(3, R, S)=6.6 .69=2484 \text {. }
$$

EXAMPLE 2. Let $r_{1}=3, r_{2}=3, r_{3}=1, s_{1}=3, s_{2}=2$, and $s_{3}=2$, then we have

and hence

$$
N_{1}=3, \quad N_{2}=3, \quad M=19,
$$

$$
H(3, R, S)=3.3 .19=171 \text {. }
$$

The corresponding number of $(0,1)$-matrices in the class $G^{\prime}$, in this example, works out to be 9 .

The above values have been verified to be correct by actual computations.

\section{$\S 5 . \quad$ Particular cases.}

In what follows we discuss a few particular cases of interest. The case I was posed to the author in a very recent communication by Professor $\mathrm{H}$. Gupta, Panjab University, Chandigarh, to whom his thanks are due; and the case II is the result obtained earlier by Anand et al. (1966) and the author (1972).

CASE I. In the preceding section if we let all the column sums $s_{i}$ 's to be 
equal to $s$, say, that is when three distinct things, each repeating $j$ times, $j \geqq 0$, are distributed among three persons, the $i$-th getting $r_{i}(\geqq 0)$, such that $r_{1}+r_{2}+r_{3}=3 s$. Then the expression (4.1) under this substitution simplifies to

$$
\begin{aligned}
M(3, R, s)= & \left(\begin{array}{c}
r_{2}+2 \\
2
\end{array}\right)\left(\begin{array}{c}
r_{3}+2 \\
2
\end{array}\right)-3\left\{\left(\begin{array}{c}
r_{2}+r_{3}-s+3 \\
4
\end{array}\right)\right. \\
& \left.-\left(\begin{array}{c}
r_{2}-s+2 \\
4
\end{array}\right)-\left(r_{3}+1\right)\left(\begin{array}{c}
r_{2}-s+1 \\
3
\end{array}\right)\right\} .
\end{aligned}
$$

CASE II. If, in case I, we further assume that all the row sums $r_{i}$ 's are equal to $r$, say, obviously $r$ and $s$ being identical. The expression (5.1), then, further reduces to

$$
\begin{aligned}
M(3, r, r)=H(3, r) & =\left(\begin{array}{c}
r+2 \\
2
\end{array}\right)^{2}-3\left(\begin{array}{c}
r+3 \\
4
\end{array}\right) \\
& =\left(\begin{array}{c}
r+2 \\
2
\end{array}\right)+3\left(\begin{array}{c}
r+3 \\
4
\end{array}\right) .
\end{aligned}
$$

AcKNowledgments. The author wishes to thank the referee for suggesting a few improvements over the original draft and for pointing out the work on $(0,1)$-matrices.

\section{Department of Mathematics \\ University of Queensland \\ St. Lucia, Brisbane Q 4067 \\ Australia}

\section{References}

[1] H. Anand, V.C. Dumir and H. Gupta, A Combinatorial Distribution Problem, Duke Math. J., 33 (1966), 757-769.

[2] W. B. Jurkat and H. J. Ryser, Term Ranks and Measurements of non-negative matrices, J. Algebra, 5 (1967), 342-357.

['3 ] K. Mano, On the formula of $H(n, r)$, Sci. Rep. Fac. Lit. Sci. Hirosaki Univ., 8 (1961), 58-60.

[4] U.S. R. Murty, Diameter of a (0,1)-matrix, Canad. Math. Bull., 11 (1968), 285288.

[5] G. B. Nath and P. V. K. Iyer, Note on the Combinatorial formula for $H(n, r), J$. Austral. Math. Soc., 14 (1972), 264-268.

[6] H. J. Ryser, Combinatorial Properties of matrices of zeros and ones, Canad. J. Math., 9 (1957), 371-377.

[7] H. J. Ryser, Matrices of zeros and ones, Bull. Amer. Math. Soc., 66 (1960a), $442-464$.

[8] H. J. Ryser, Traces of matrices of zeros and ones, Canad. J. Math., 12 (1960b), $463-476$. 